Supplementary Materials for

\title{
Ionic-covalent hybrid tough hydrogels enabled by the in situ release of metal ions from insoluble salts or alkalis
}

Chun Li, Xiaohu Zhou, 'Dan Zhou, Fan Chen, Jiayan Shen, Huifang Li, Jie Zhang, and Xuechang Zhou*

College of Chemistry and Environmental Engineering, Shenzhen University, Shenzhen

518060, P. R. China

*Correspondence and requests for materials should be addressed to X. H. Z. (email:

xhzhou@szu.edu.cn) or X. C. Z. (email: $\underline{x c z h o u @ s z u . e d u . c n)})$. 


\section{Experimental Section}

\section{Materials:}

Sodium alginate, cobalt hydroxide $\left[\mathrm{Co}(\mathrm{OH})_{2}\right]$, barium carbonate $\left(\mathrm{BaCO}_{3}\right)$, lanthanum carbonate $\left[\mathrm{La}_{2}\left(\mathrm{CO}_{3}\right)_{3} \times \mathrm{xH}_{2} \mathrm{O}\right]$ were purchased from Aladdin. Acrylamide (AAm), ammonium persulfate (APS), calcium carbonate $\left(\mathrm{CaCO}_{3}\right)$, copper hydroxide $\left[\mathrm{Cu}(\mathrm{OH})_{2}\right]$ and $\mathrm{D}-(+)$-Gluconic acid $\delta$-lactone (GDL) were purchased from Macklin. $\mathrm{N}, \mathrm{N}^{\prime}$-Methylenebisacrylamide (MBAA) and $\mathrm{N}, \mathrm{N}, \mathrm{N}^{\prime}, \mathrm{N}^{\prime}$-tetramethylethylenediamine (TEMED) were purchased from Sigma-Aldrich.

\section{Ca-alginate/PAAm hydrogel synthesis}

$\mathrm{CaCO}_{3}(0.05 \mathrm{~g})$ was ultrasonically dispersed in $25 \mathrm{~mL}$ deionized water. Sodium alginate $(0.50 \mathrm{~g})$ and acrylamide (AAm, $4.00 \mathrm{~g})$ were dissolved completely to the above turbid solution for $4 \mathrm{~h}$. After degassing for 10 minutes in vacuum, $120 \mu \mathrm{L} 0.10 \mathrm{~g} / \mathrm{mL}$ ammonium persulphate (APS) aqueous solution as photothermal initiator, $192 \mu \mathrm{L} 0.025$ $\mathrm{g} / \mathrm{mL} \mathrm{N}, \mathrm{N}^{\prime}$-methylenebisacrylamide (MBAA) aqueous solution as crosslinking agent, and $20 \mu \mathrm{L}$ N,N,N',N'-tetramethylethylenediamine (TEMED) as crosslinking accelerator were added to form the precursor solution. In addition, D-(+)-Gluconic acid $\delta$-lactone (GDL) was added to the precursor solution. Subsequently, the resulting solution was poured about $3 \mathrm{~g}$ into a rectangular mould measuring $23 \times 43 \times 19 \mathrm{~mm}^{3}$ and cured with UV light at a wavelength of $254 \mathrm{~nm}\left(25 \mathrm{~W}, \mathrm{ZF}-5\right.$, ShanghaiJiaPeng) at $40{ }^{\circ} \mathrm{C}$ for $1 \mathrm{~h}$. The cured hybrid gel was left in a humid box for 24 hours to stabilize the reactions.

\section{$\mathbf{M}^{\mathrm{n}+}$-alginate/PAAm hydrogel synthesis}

Sodium alginate $(0.50 \mathrm{~g})$ was dissolved to $16 \mathrm{~mL}$ deionized water for $4 \mathrm{~h}$ and then blended with another prepared solution (4.00 g AAm, insoluble salt or alkali, $192 \mu \mathrm{L}$ $0.025 \mathrm{~g} / \mathrm{mL}$ MBAA, $120 \mu \mathrm{L} 0.1 \mathrm{~g} / \mathrm{mL}$ APS). After stirring for 10 minutes, added GDL and $20 \mu \mathrm{L}$ TEMED to synthesize the $\mathrm{M}^{\mathrm{n}+}$-alginate/PAAm hybrid gels. The usage of substances was summarized in Table S1. 


\section{pH measurement}

The metal salts or alkalis and GDL were dissolved in $25 \mathrm{~mL}$ deionized water for $24 \mathrm{~h}$, and the $\mathrm{pH}$ of the prepared transparent solutions was measured with the calibrated $\mathrm{pH}$ meter (LE438 FE28-Standa) (Table S1).

Table S1. The protocol of the $\mathrm{M}^{\mathrm{n}+}$-alginate/PAAm hybrid gels and the $\mathrm{pH}$ of the corresponding reaction solutions.

\begin{tabular}{|c|c|c|c|c|c|}
\hline Metal ion source & $\mathrm{c}\left(\mathrm{M}^{\mathrm{n}+}\right)(\mathrm{mM})$ & $\mathrm{m}(\mathrm{mg})$ & $\mathrm{m}(\mathrm{GDL})(\mathrm{g})$ & $\mathrm{GDL} \mathrm{M}^{\mathrm{n}+}$ in mole & $\mathrm{pH}$ \\
\hline $\mathrm{CaCO}_{3}$ & 12 & 30.0 & 0.107 & $2: 1$ & 6.44 \\
\hline $\mathrm{BaCO}_{3}$ & 6 & 29.6 & 0.053 & $2: 1$ & 6.23 \\
\hline $\mathrm{Co}(\mathrm{OH})_{2}$ & 12 & 27.9 & 0.107 & $2: 1$ & 5.34 \\
\hline $\mathrm{Cu}(\mathrm{OH})_{2}$ & 12 & 29.3 & 0.107 & $2: 1$ & 4.14 \\
\hline $\mathrm{La}_{2}\left(\mathrm{CO}_{3}\right)_{3} \cdot \mathrm{xH}_{2} \mathrm{O}$ & 12 & 68.7 & 0.160 & $3: 1$ & 3.74 \\
\hline
\end{tabular}

The different ratio of $\mathrm{CaCO}_{3}$ and GDL in mole were dissolved in $25 \mathrm{~mL}$ deionized water for $24 \mathrm{~h}$, and the $\mathrm{pH}$ of the prepared transparent solutions was measured with the calibrated pH meter (LE438 FE28-Standa) (Table S2).

Table S2. The protocol of the different ratios of $\mathrm{GDL} / \mathrm{CaCO}_{3}$ in mole of Ca-alginate/PAAm hydrogel and the $\mathrm{pH}$ of the corresponding reaction solutions.

\begin{tabular}{|c|c|c|c|c|}
\hline $\mathrm{GDL} / \mathrm{CaCO}_{3}$ in mole & $\mathrm{c}\left(\mathrm{M}^{\mathrm{n}+}\right)(\mathrm{mM})$ & $\mathrm{c}(\mathrm{GDL})$ & $\mathrm{m}(\mathrm{GDL})(\mathrm{mg})$ & $\mathrm{pH}$ \\
\hline $0.5: 1$ & 20 & 10 & 45 & 7.47 \\
\hline $1.0: 1$ & 20 & 20 & 89 & 7.18 \\
\hline $1.5: 1$ & 20 & 30 & 134 & 6.79 \\
\hline $2.0: 1$ & 20 & 40 & 178 & 6.44 \\
\hline $3.0: 1$ & 20 & 60 & 267 & 3.85 \\
\hline
\end{tabular}

\section{Rheological investigation of Ca-alginate/PAAm hydrogel}

Rheological investigation of the precursor solution was conducted with the rheometer (AR 1000, TA Instruments) under $1 \%$ of strain and $10 \mathrm{rad} / \mathrm{s}$ of angular frequency for 2000 seconds at room temperature. The distance between the clamps was constant at 1.5 
mm.

\section{Mechanical properties of Ca-alginate/PAAm hydrogel}

For all stress-strain tensile tests, the hydrogel samples (gauge width: $23 \pm 0.5 \mathrm{~mm}$, gauge length: $20 \pm 1 \mathrm{~mm}$, thickness: $2.5 \pm 0.2 \mathrm{~mm}$ ) were stretched uniaxial at a velocity of $40 \mathrm{~mm} / \mathrm{min}$ until fracture with the tensile testing machine (CMT6103, SANS). For tensile recovery tests, the Ca-alginate/PAAm hydrogel $(20 \mathrm{mM})$ were stretched to $600 \%$ strain and then immediately unloaded to their original length. The stretched samples were self-recovered at $80{ }^{\circ} \mathrm{C}$ for $10 \mathrm{~s}, 10 \mathrm{~min}, 1 \mathrm{~h}, 24 \mathrm{~h}$. Subsequently, the sampled were cooled to room temperature and then tested with another $600 \%$ stretch. For hysteresis tests, the Ca-alginate/PAAm hydrogel $(20 \mathrm{mM})$ were stretched to $300 \%, 600 \%, 900 \%$, $1200 \%$ strain respectively and then immediately unloaded to their original length to obtain the loading-unloading curve.

The Elastic modulus was determined from the slope of the stress-strain curve at strain between $0 \sim 10 \%$. The fracture energy was calculated from $\Gamma=\frac{U\left(L_{c}\right)}{a_{0} b_{0}} . L_{c}$ was the length of the gel at fracture strain, $U\left(L_{c}\right)$ was the area beneath the force-displacement curve from the distance between $0 \sim L_{c}$, and $a_{0}$ and $b_{0}$ were the width and thickness of the sample, respectively.

\section{The Homogeneity tests of the Ca-alginate/PAAm hydrogel}

Each sample was cut into seven pieces evenly, and the weight of each piece was determined. After frozen-dried for $24 \mathrm{~h}$, the dried weight of the samples was determined again, and the water content of each piece was calculated (Figure S2).

The hydrogel sample was cut into small pieces. After frozen-dried for $48 \mathrm{~h}$ by lyophilizer (SCIENTZ-12N), Scanning Electronic Microscopy (SEM, S-3400N, HITACHI) and Energy Dispersive Spectrometer (EDS, S-3400N, HITACHI) test were carry out, the SEM image and line scan of EDS is shown in the Figure S3-4. 
S-5 


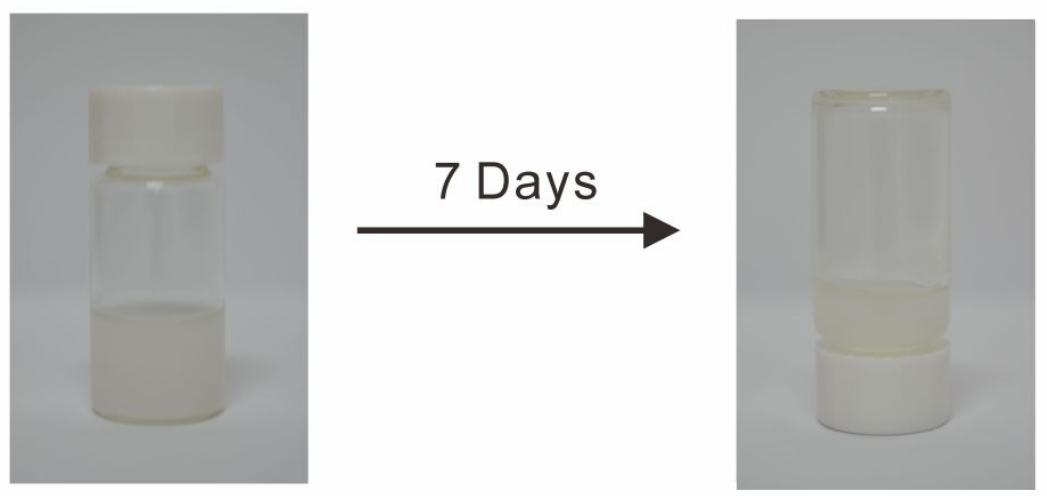

Figure S1. The sodium alginate/acrylamide precursor solution could be stored for seven days without gelation.

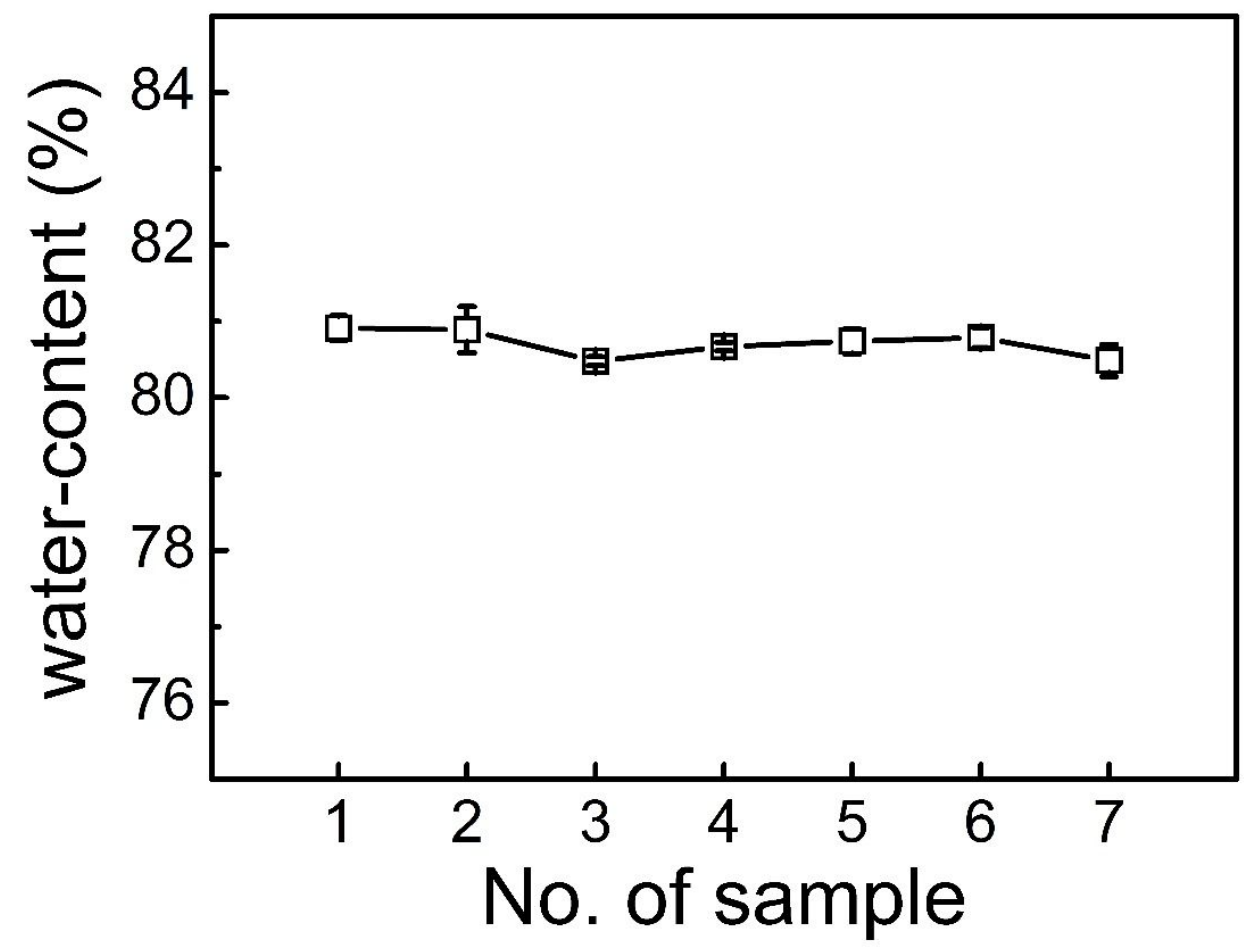

Figure S2. The sliced samples with similar water content confirmed the homogeneity of the Ca-alginate/PAAm hybrid gel. 

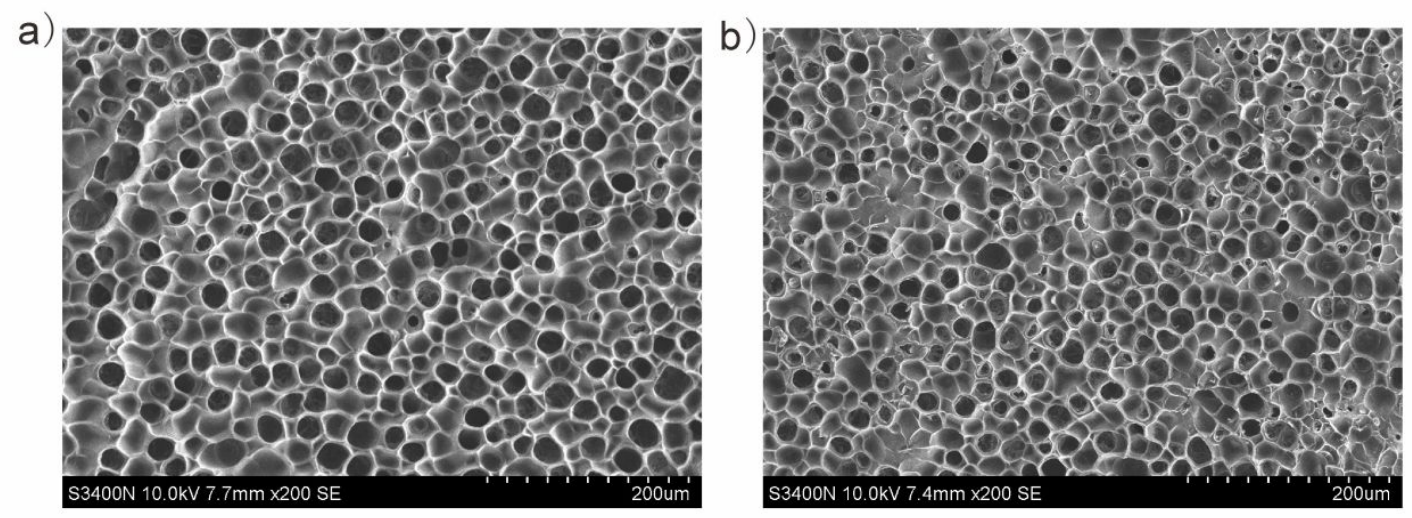

Figure S3. a, b) The SEM images of two different $20 \mathrm{mM} \mathrm{Ca}$-alginate/PAAm samples. The homogeneous porous structure of each sample indicated the homogeneity of the hybrid gel, and the similar porous structure between the samples indicated the robustness of this synthesis method.

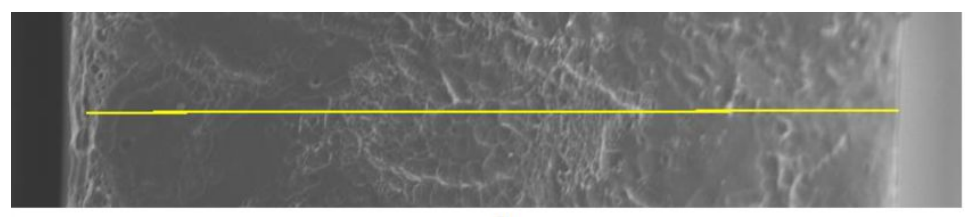

$\mathrm{Ca}_{\mathbf{a}}$

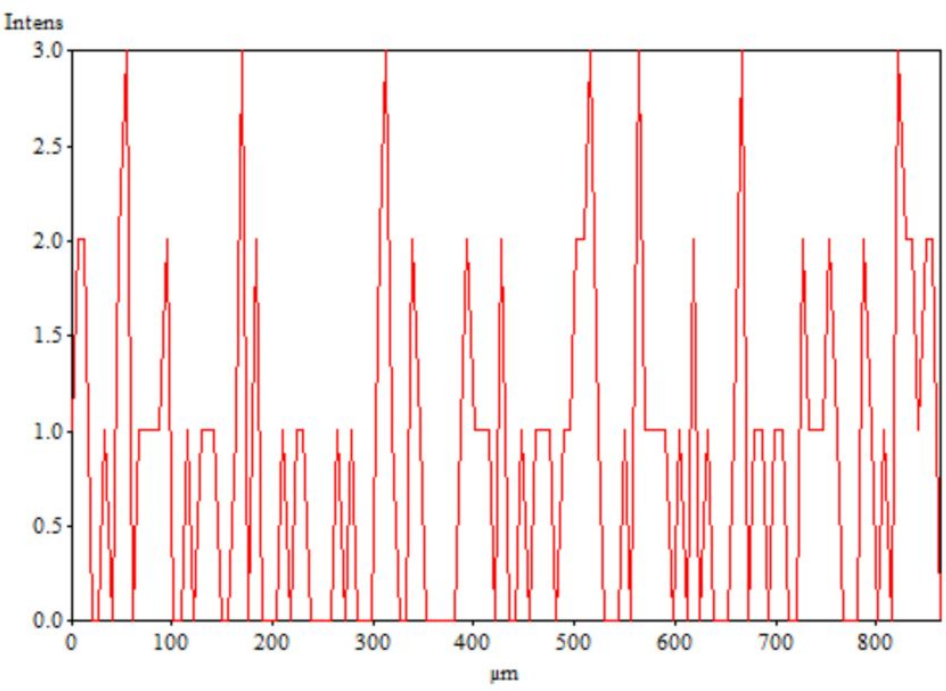

Figure S4. SEM-EDS profile showed the concentration distribution of the calcium ion in the hybrid gel from the top surface to the bottom, and the uniform distribution confirmed the homogeneity of the Ca-alginate/PAAm hybrid gel. 


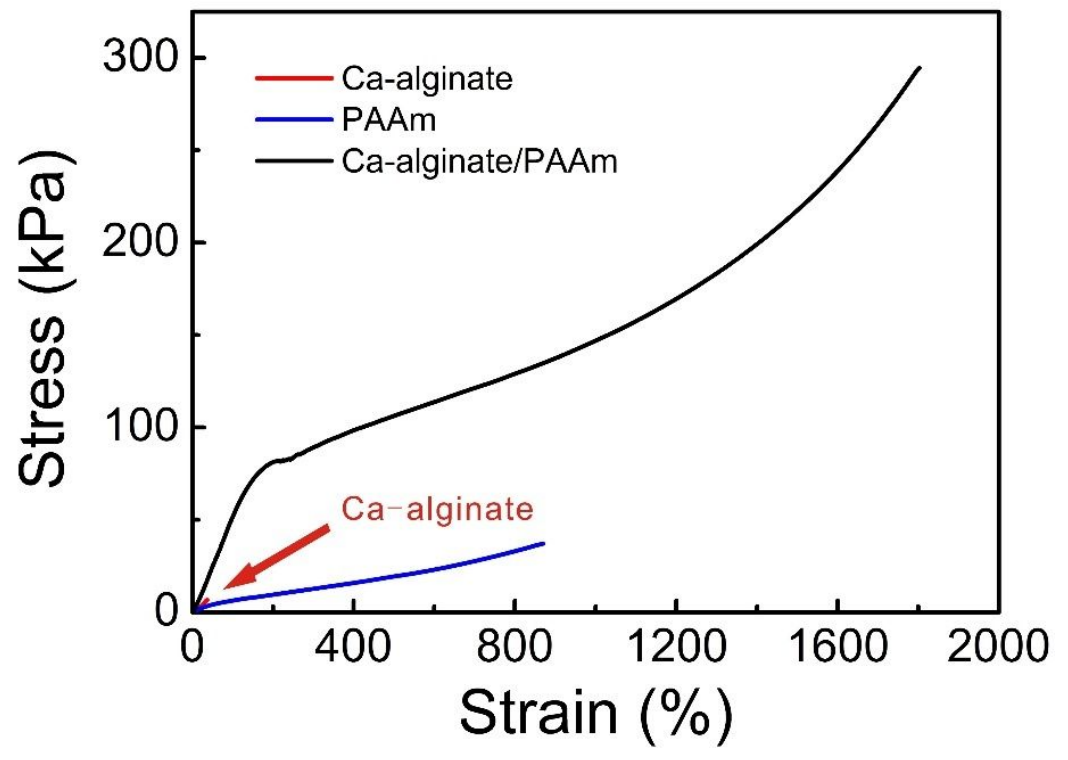

Figure S5. Stress-strain curves of the calcium alginate gel (1.7 wt \% sodium alginate with $20 \mathrm{mM} \mathrm{Ca}^{2+}$ ), PAAm gel (13.6 wt \% acrylamide) and calcium alginate/PAAm hybrid gel (1.7 wt \% sodium alginate with $20 \mathrm{mM} \mathrm{Ca}^{2+}$ and $13.6 \mathrm{wt} \%$ acrylamide). 